\title{
Comparative ultrastructural study of human corpus cavernosum during ageing
}

\author{
N. Tomada ${ }^{*}$, R. Oliveira ${ }^{*}$, I. Tomada $^{* *}$, P. Vendeira ${ }^{*}, \mathrm{D}$. Neves $^{* * *}$ \\ * Department of Urology of S. João Central Hospital, Porto - 4200-319 Porto - Portugal \\ ** Master Student of Faculty of Nutrition and Food Sciences of Universidade do Porto \\ (FCNAUP) - 4200-465 Porto - Portugal \\ ${ }^{* * *}$ Laboratory of Molecular Cell Biology of Faculty of Medicine and IBMC of Universidade \\ do Porto - 4200-319 Porto - Portugal; The first 2 authors had the same contribution to the \\ presented work \\ nunotomada@gmail.com
}

Erectile Dysfunction (ED), the inability to achieve or maintain an erection of sufficient rigidity for completion of sexual act, is a common condition affecting more than 150 million of men worldwide [1]. This disorder is highly associated with aging, however concomitant pathologies such as hyperlipidemia, hypertension, and diabetes also contribute to ED progression. In the Massachusetts Male Aging Study, age was considered an independent variable strongly associated with ED, showing that the prevalence of this disease increased with age from $38 \%$ in the youngest group of men (mean age $40 \mathrm{y}$.) to almost $70 \%$ in the oldest group of men examined (mean age 70y.) [2]. It is well demonstrated that aging leads to changes in the cardiovascular system, which results in a decrease in elasticity due to fibrosis and an increase in stiffness of the arterial system, independently of the effects of concurrent pathologies [3]. Vasculogenic ED is the most prevalent condition, affecting nearly $80 \%$ of patients with organic etiology [4]. Small vessels of the penis are very sensitive to structural and functional changes, and small disturbances can conduce to ED [5]. ED is now considered by some authors as synonymous to endothelial dysfunction and an early manifestation of atherosclerosis, being a precursor of systemic vascular disease [6-8]. Human cavernous tissue is mainly constituted by smooth muscle fibers that surround sinusoid vessels. Corpus cavernosum structural elements act in concert, allowing increase of intra-cavernous arterial flow and smooth muscle relaxation processes which are fundamental to penile erection. The aim of this study was to compare the ultrastructural anatomy of the young and aged human corpus cavernosum, in the absence of additional risk factors.

The human penile fragments used in this study were removed from patients submitted to penile deviation surgery (11 cases) (age varying from 60-70 years) and from potential organ donors (4 cases) (age varying from 18-28 years) without known ED risk factors. The local hospital and university ethics committee authorized the study design. The tissue was fixed in $2.5 \%$ glutaraldehyde with $5 \mathrm{mM} \mathrm{CaCl} 2$, in $0.1 \mathrm{M}$ cacodylate buffer, $\mathrm{pH} 7.2$, for $2 \mathrm{~h}$, rinsed in buffer with $7 \%$ sucrose overnight, post-fixed in $1 \%$ osmium tetroxide in cacodylate buffer, $\mathrm{pH} 7.2$ at room temperature, for $1 \mathrm{~h}$, dehydrated in ascending concentrations of ethanol, and embedded in Epon. Ultrathin sections were stained with aqueous uranyl acetate for $5 \mathrm{~min}$, lead citrate for $5 \mathrm{~min}$ and observed in Jeol 100B electron microscope (Jeol Ltd. Tokyo, Japan). Morphometric study was performed in random selected 16 micrographs. Total areas of endothelium, smooth muscle, collagen and connective tissue cells (mainly fibroblasts) were quantified. Statistical analysis was done employing absolute values using Statistical Package for the Social Sciences (SPSS), version 
13.0 for Windows (SPSS Inc., IL, USA) and the difference of mean values between groups was assessed by two-tail $t$ test. A value of $P<0.05$ was considered significant.

All samples of corpus cavernosum presented well preserved endothelium (Fig. 1a and 1b) and profuse smooth muscle cells (SMC) surrounded by basal lamina (Fig.1c and d). No marked differences were seen between basal lamina thickness in the studied samples, neither in the subendothelial face (Fig. $1 \mathrm{a}$ and b) nor in the surrounding SMC (Fig 1c and d). Connective tissue consisting mainly of fibroblasts and fibres of homogeneous diameter organized in small bundles structurally resembling collagen fibrils spread out between SMC fibres. Samples recovered from older patients (Fig. $1 \mathrm{~b}$ and d) presented an increase in collagen fibrils deposition, and SMC exhibited poorer organized cytoskeleton when compared with that from younger tissue (Fig. 1a and c). Morphometric assay revealed that the jointly percentage of SMC and connective tissue, including fibroblasts was higher than $80 \%$ both in corpus cavernosum samples obtained from young and aged patients (Fig.2). However, marked differences were observed in the ratio of these two cell populations during ageing. SMC percentage decrease significantly and connective tissue components (collagen and fibroblasts) increase in the same proportion in aged tissue (Fig. 2).

Access to corpus cavernosum tissue from patients has been a major impediment of studies in human, so we often needed to employ animal models with appropriate risk factors and pathologies. In the present work we used cavernous tissues obtained from patients without any known risk factor for ED, harvested from programmed surgeries or potential organ donors. In this way, we considered that ageing was the only variable between samples employed in the morphometric study. Aging male reproductive tract is characterized by a variable degree of tissue remodelling malfunction of the corpora cavernosa [9]. The increase in collagen fibers deposition and significant decrease in SMC, and consequently enhanced stiffness of the fibro-elastic elements of the cavernosous tissues, observed in our study may explain the slow kinetics of both erection and detumescence. Previous studies in experimental models of ageing rat showed an increase in the apoptotic index in the penile trabecular and arterial smooth muscle [10]. This issue together with the increase in fibrosis in the erectile tissue may explain the loss in corpora cavernosal compliance. On the other hand, SMC homeostasis, as well as eNOS and PDE-5 expression are also strongly dependent on the presence of androgens, and ageing is associated with a decrease in bioavailable testosterone. However, androgen monotherapy is unable to reverse ED probably due to the multifactorial nature of the disease [11]. In the same way, aging in otherwise healthy patients is associated with reduction in NO levels from eNOS downregulation due endothelial damage, excessive release of adrenergic compounds through endothelin and rho kinase activation, and/or impairment of SMC relaxation by endogenous factors [12]. Characterization of the ultrastructural changes of cavernous tissue would be relevant in the elucidation of ageing specific contribution to erectile dysfunction onset. Nevertheless, further molecular studies will be approached in human corpus cavernosum samples in order to clarify ageing-related mechanisms of ED. 


\section{References}

[1] M. Braun et al., Int. J. Impot. Res. 12 (2000) 305.

[2] H. Feldman et al., J. Urol. 151 (1994) 54.

[3] M. Cheitlin, Am. J. Geriatr. Cardiol. 12 (2003) 9.

[4] H. Brunner et al., J. Hypert. 23 (2005) 233.

[5] C. Kaya et al., Int. J. Impot. Res. 18 (2006) 55.

[6] M. Cheitlin, J. Am. Coll. Cardiol. 43 (2004) 185.

[7] I. Goldstein, Int. J. Impot. Res. 15 (2003) 229.

[8] A. Guay, Endocrinol. Metab. Clin. N. Am. 36 (2007) 453.

[9] E. Wespes, Curr. Opin. Urol. 10 (2000) 625.

[10] M. Ferrini et al., Biol. Reprod. 64 (2001) 974.

[11] N. Mikhail, Am. J. Med. 119 (2006) 373.

[12] N. Gonzalez-Cadavid, J. Raifer, Exp. Gerontol. 39 (2004) 1705.
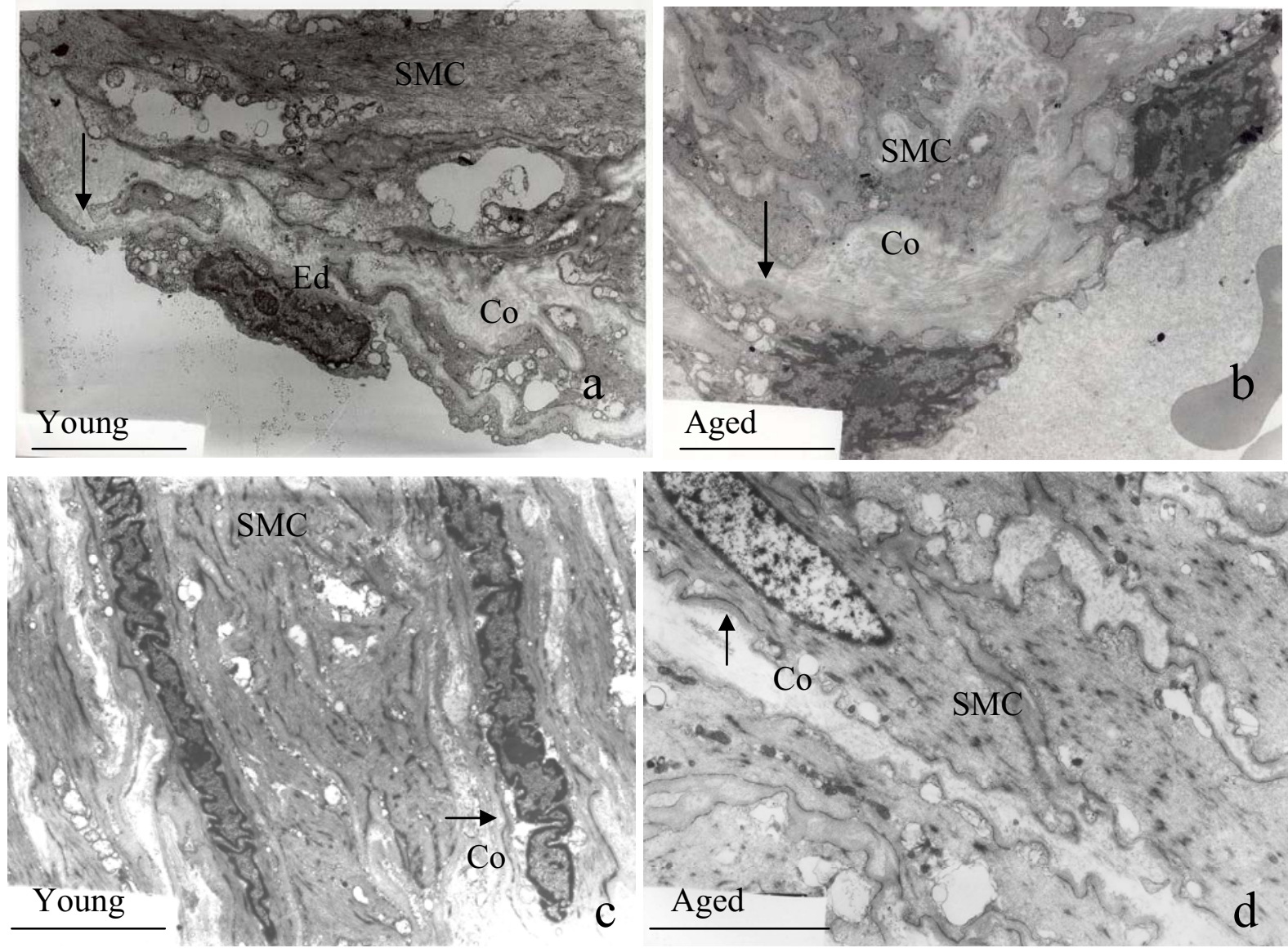

Fig. 1. Corpus cavernosum micrographs from young (a and c) and aged ( $b$ and d) patients. Note Endothelium (Ed), smooth muscle cells (SMC), collagen (Co) deposition in connective tissue and basal lamina (arrow) Scale bar - $5 \mu \mathrm{m}$ 


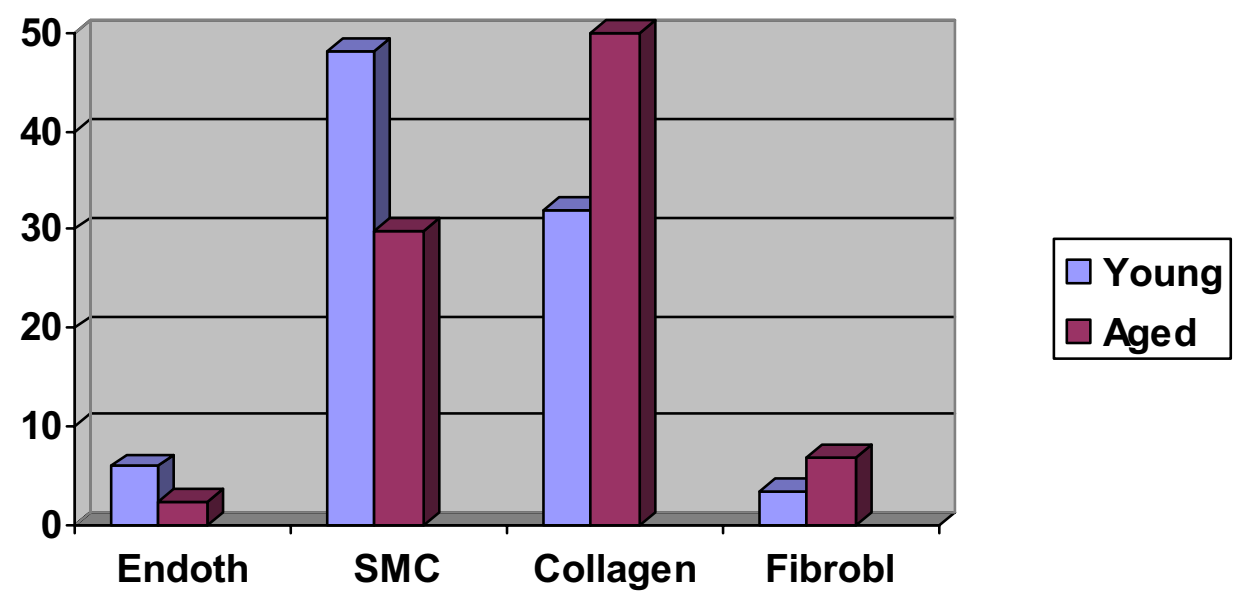

Fig.2. Comparative percentage distribution of cellular constitution of young and aged human corpus cavernosum. Endothelium (endoth), smooth muscle cells (SMC), collagen (col) and fibroblasts (fibrobl). All the parameters measured present significant variation $(\mathrm{P}<0.05)$ between young and aged samples. 